\title{
The evaporation residue in the fission state of Barium nuclei within relativistic mean-field theory
}

\author{
M. Bhuyan ${ }^{1}$, S. K. Patra ${ }^{1}$ and Raj K. Gupta ${ }^{2}$ \\ ${ }^{1}$ Institute of Physics, Sachivalaya Marg, Bhubaneswar- 751 005, India. \\ ${ }^{2}$ Department of Physics, Panjab University, Chandigarh- 160 014, India *
}

(Dated: June 14, 2021)

\begin{abstract}
The evaporation residue of Barium isotopes are investigated in a microscopic study using relativistic mean field theory. The investigation includes the isotopes of Barium from the valley of stability to exotic proton-rich region. The ground as well as neck configurations for these nuclei are generated from their total nucleonic density distributions of the corresponding state. We have estimated the constituents (number of nucleons) in the elongated neck region of the fission state. We found the $\alpha$-particle as the constituent of neck of Ba-isotopes, referred to as the evaporated residue in heavy-ion reaction studies. A strong correlation between the neutron and proton is observed throughout the isotopic chain.

PACS numbers: 21.10.Dr., 21.60.-n., 23.60.+e., 24.10.Jv.
\end{abstract}

\section{INTRODUCTION}

In atomic nuclei, the occurrence of clusters and their cluster structures were already predicted in late 1930's by Wheeler and von Weizacker [1, 2]. Numerous experimental and theoretical studies based on advanced microscopic approaches with single-nucleon degrees of freedom have revealed a wealth of data on clustering phenomena in light nuclei [3-8]. Another instance of clustering in atomic nuclei is the cluster radioactivity, first predicted theoretically [9, 10], based on quantum mechanical fragmentation theory [11-13], and then observed experimentally [14]. Few other testimony for the formation of nuclear clustering are reported from experiments, but indirectly. The most possible clusters in the ground and excited states of light and medium mass nuclei are the $N=Z{ }^{4} \mathrm{He},{ }^{8} \mathrm{Be},{ }^{12} \mathrm{C},{ }^{16} \mathrm{O},{ }^{20} \mathrm{Ne},{ }^{24} \mathrm{Mg}$ and ${ }^{28} \mathrm{Si}$ nuclei [4, 15,-19].

Clustering is the essential feature of many-nucleon dynamics that coexists with the nuclear mean-field. In this context, one can say that a fully microscopic understanding and description of cluster formation and their emission is necessary in mean-field approaches, despite the existence of clusterization effects in many calculations [20-22]. This is because, in most of these calculations, we are neglecting the structure effects such as the binding energies and scattering phase shifts of these configurations that are assumed as a-priory ingredients of effective interactions in the mean-field models [2325]. Another consequence is that the nuclear deformation in ground state plays an important role in the formation of a cluster because it removes the degeneracy of single nucleon levels associated with spherical symmetry. At some specific deformation, the restored degeneracy degenerates the deformed shell closures and facilitates the formation of clusters. However, this is a rather qualitative explanation because the clustering phenomenon can not generally be explained by accidental degeneracies. Hence, in order to understood the mechanism of clustering, a more general description that encompasses both cluster and quantum liquid-drop aspects in the fi- nite nuclear systems are needed.

The aim of this work is to address the origin of neck, assumed to be required for the preformed clusters in the fission state, i.e., the formation of elongated neck in the fission state of a nucleus. It is worth mentioning that the relativistic meanfield approach is able to give a complete and accurate description of ground-state properties and collective excitations over the whole nuclear chart including the superheavy mass regions [20, 23, 24]. In this approach, the many-body dynamics is represented by independent nucleons interacting through the meson field with a local self-consistent mean-field potential that corresponds to the actual density and current distribution of a given nucleus for a given state. In a recent work [20], we have explained the formation of cluster(s) and their composition in the ground and intrinsic excited state(s) of Ba isotopes. In the present study, we are interested to reveal the fission state of Ba nuclei from a microscopic description based on the framework of relativistic mean field theory. The most important steps that have been taken here are to find the constituents or the composition of neck, which plays an important role for the explanation of a fission state. In a fission process, the neck is well accepted to be the nucleon's emission region [20, 21]. It will truly be of benefit if it would be possible to generate the neck structure theoretically and find out the composition of this region quantitatively. Such a study will be accessible for understanding of the fission state as well as its residue in an heavy-ion collision experiment where the excited compound nucleus decays by the emission of light-particle evaporation residues and the fission fragments. For $\mathrm{Ba}^{*}$ compound systems (specifically, ${ }^{118,122} \mathrm{Ba}^{*}$ ), though the charge distribution for fission fragments with $3 \leq \mathrm{Z} \leq 28$ is measured [26, 27], only the total cross-section $\sigma_{E R}$ is measured for the evaporation residues (ER: $Z<3)$ ), but without identifying the constituents ( $\mathrm{Z}$ or A-value) of $\sigma_{E R}$. It is, therefore, of interest to know the constituent(s) or the particle(s) in the neck region of the fission state of Ba nuclei, in the RMF formalism - a structure calculation. These constituent(s) will belong to ER or fission, depending on the charge number (Z-value) of the particle(s) in neck region is less than or greater than $Z=2$.

The paper is organized as follows: Section II gives a brief description of the relativistic mean-field formalism (RMF). The effects of pairing for open shell nuclei, included in our 
calculations, are also discussed in this section. The results of our calculations are presented in Section III. The neck configuration and their composition are also discussed quantitatively in this section. A summary of the results obtained, together with concluding remarks, are given in the last Section IV.

\section{THE RELATIVISTIC MEAN-FIELD THEORY}

In the last few decades, the relativistic mean field theory is applied successfully to study the structural properties of nuclei throughout the nuclear chart, including also the unknown island of superheavy nuclei [23, 28,-33]. The relativistic Lagrangian density for nucleon-meson many-body system is expressed as [31, 33],

$$
\begin{aligned}
\mathcal{L}= & \bar{\psi}_{i}\left\{i \gamma^{\mu} \partial_{\mu}-M\right\} \psi_{i}+\frac{1}{2} \partial^{\mu} \sigma \partial_{\mu} \sigma-\frac{1}{2} m_{\sigma}^{2} \sigma^{2} \\
& -\frac{1}{3} g_{2} \sigma^{3}-\frac{1}{4} g_{3} \sigma^{4}-g_{s} \overline{\psi_{i}} \psi_{i} \sigma-\frac{1}{4} \Omega^{\mu \nu} \Omega_{\mu \nu} \\
& +\frac{1}{2} m_{w}^{2} V^{\mu} V_{\mu}+\frac{1}{4} c_{3}\left(V_{\mu} V^{\mu}\right)^{2}-g_{w} \bar{\psi}_{i} \gamma^{\mu} \psi_{i} V_{\mu} \\
& -\frac{1}{4} \vec{B}^{\mu \nu} \cdot \vec{B}_{\mu \nu}+\frac{1}{2} m_{\rho}^{2} \vec{R}^{\mu} \cdot \vec{R}_{\mu}-g_{\rho} \bar{\psi}_{i} \gamma^{\mu} \vec{\tau} \psi_{i} \cdot \vec{R}^{\mu} \\
& -\frac{1}{4} F^{\mu \nu} F_{\mu \nu}-e \bar{\psi}_{i} \gamma^{\mu} \frac{\left(1-\tau_{3 i}\right)}{2} \psi_{i} A_{\mu} .
\end{aligned}
$$

All the quantities in the above Lagrangian density have their usual well known meanings. From the Lagrangian we obtain the field equations for the nucleons and mesons. These equations are solved by expanding the upper and lower components of the Dirac spinors and the boson fields in an axially deformed harmonic oscillator basis with an initial deformation $\beta_{0}$. The set of coupled equations is solved numerically by a self-consistent iteration method. The center-of-mass motion energy correction is estimated by the usual harmonic oscillator formula $E_{c . m} .=\frac{3}{4}\left(41 A^{-1 / 3}\right)$. The quadrupole deformation parameter $\beta_{2}$ is evaluated from the resulting proton and neutron quadrupole moments, as $Q=Q_{n}+Q_{p}=$ $\sqrt{\frac{16 \pi}{5}}\left(\frac{3}{4 \pi} A R^{2} \beta_{2}\right)$. The root mean square (rms) matter radius is defined as $\left\langle r_{m}^{2}\right\rangle=\frac{1}{A} \int \rho\left(r_{\perp}, z\right) r^{2} d \tau$, where $A$ is the mass number, and $\rho\left(r_{\perp}, z\right)$ is the deformed density. The total binding energy and other observables are also obtained by using the standard relations, given in Ref. [34]. We use the well known NL3 parameter set [35], which not only reproduces the properties of stable nuclei but also well predicts for those nuclei far from the $\beta$-stability valley. As outputs, we obtain different potentials, densities, single-particle energy levels, radii, deformations and the binding energies. For a given nucleus, the maximum binding energy corresponds to the ground state and other solutions are obtained as various excited intrinsic states, provided the nucleus does not undergo fission. For the fission state [36-38], we follow a special procedure discussed below.

\section{A. Pairing Effect}

In the present study, we are dealing with the proton-rich isotopes of Ba nucleus, hence pairing is a crucial quantity for the open shell nuclei in determining the nuclear gross properties. The constant gap, BCS-pairing approach has been adopted for the present calculations. The general expression for pairing energy is given as:

$$
E_{\text {pair }}=-G\left[\sum_{i>0} u_{i} v_{i}\right]^{2}
$$

where $G$ is the pairing force constant and $v_{i}^{2}$ and $u_{i}^{2}=1-v_{i}^{2}$ are the occupation probabilities. The variational procedure with respect to the occupation numbers $v_{i}^{2}$, gives the BCS equation $2 \epsilon_{i} u_{i} v_{i}-\triangle\left(u_{i}^{2}-v_{i}^{2}\right)=0$ with $\triangle=G \sum_{i>0} u_{i} v_{i}$. This is the famous BCS equation for pairing energy. The densities are contained within the occupation number $n_{i}=v_{i}^{2}=$ $\frac{1}{2}\left[1-\frac{\epsilon_{i}-\lambda}{\sqrt{\left(\epsilon_{i}-\lambda\right)^{2}+\triangle^{2}}}\right]$. In order to take care of the pairing effects in the present study, we use the constant gap for proton and neutron, as given in [39]: $\triangle_{p}=R B_{s} e^{s I-t I^{2}} / Z^{1 / 3}$ and $\triangle_{n}=R B_{s} e^{-s I-t I^{2}} / A^{1 / 3}$, with $R=5.72, s=0.118, t=$ 8.12, $B_{s}=1$, and $I=(N-Z) /(N+Z)$. (Note that the gaps obtained by these expressions are valid for nuclei both on or away from $\beta$-stable region.)

In solving the RMF equations, the pairing force constant $G$ is not calculated explicitly. Instead, using the above gap parameter, we calculate directly the occupation probability using the chemical potentials for nucleons $\lambda_{n}$ and $\lambda_{p}$. Finally, we can write the pairing energy as:

$$
E_{\text {pair }}=-\triangle \sum_{i>0} u_{i} v_{i}^{2}
$$

Apparently, in a given nucleus, for a constant pairing gap $\triangle$, the pairing energy $E_{\text {pair }}$ is not constant since it depends on the occupation probabilities $v_{i}^{2}$ and $u_{i}^{2}$, and the deformation parameter $\beta_{2}$. For example, for a constant pairing parameter $\triangle$ and force constant $G$, the pairing energy $E_{\text {pair }}$ diverges if it is extended to an infinite configuration space. In fact, in all realistic calculations with finite range forces, $\triangle$ decreases with state (spherical or deformed) for large momenta near the Fermi surface. However, in the present case, we assume that pairing gap for all states $|\alpha>=| n l j m>$ are equal to each other near the Fermi surface and hence a constant pairing gap is taken for simplicity of the calculations. We use in our calculations a pairing window, and all the equations extended up to the level $\epsilon_{i}-\lambda \leq 2\left(41 A^{1 / 3}\right)$. The factor 2 has been determined so as to reproduce the pairing correlation energy for neutrons in ${ }^{118} \mathrm{Sn}$ using Gogny force [34]. This type of prescription for pairing effects, both in RMF and Skyrme Hartree Fock (SHF), has already been used by us and many others authors [20, 40]. Within this pairing approach, it is shown [40, 41] that the results for binding energies and quadruple deformations are almost identical with the predictions of relativistic Hartree-Bogoliubov (RHB) approach [41, 42]. 
TABLE I: The RMF(NL3) results for binding energy, pairing energy, the charge radii and quadrupole deformation parameter $\beta_{2}$ for ${ }^{112-134} \mathrm{Ba}$ nuclei for the ground-state and fission state, compared with the FRDM predictions [43, 44] and the experimental data [45. 47]. The energy in $\mathrm{MeV}$ and radius in $\mathrm{fm}$.

\begin{tabular}{|c|c|c|c|c|c|c|c|c|c|c|}
\hline \multirow[t]{2}{*}{ Nucleus } & \multicolumn{3}{|c|}{ Binding Energy } & \multirow{2}{*}{$\begin{array}{l}\text { Pairing Energy } \\
E_{\text {pair }}\end{array}$} & \multicolumn{3}{|c|}{ Charge Radius } & \multicolumn{3}{|c|}{ Deformation Parameter } \\
\hline & NL3 & Expt. & FRDM & & NL3 & Expt. & FRDM & NL3 & Expt. & FRDM \\
\hline \multirow[t]{2}{*}{${ }^{112} \mathrm{Ba}$} & 895.33 & & 894.88 & 21.43 & 4.754 & & & 0.239 & & 0.207 \\
\hline & 857.12 & & & 19.35 & 9.251 & & & 5.562 & & \\
\hline \multirow[t]{2}{*}{${ }^{114} \mathrm{Ba}$} & 920.67 & 922.26 & 921.26 & 21.29 & 4.765 & & & 0.235 & & 0.243 \\
\hline & 880.37 & & & 19.85 & 9.252 & & & 5.561 & & \\
\hline \multirow[t]{2}{*}{${ }^{116} \mathrm{Ba}$} & 947.55 & 947.14 & 946.85 & 20.82 & 4.786 & & & 0.294 & & 0.280 \\
\hline & 902.14 & & & 20.15 & 9.255 & & & 5.564 & & \\
\hline \multirow[t]{2}{*}{${ }^{118} \mathrm{Ba}$} & 971.43 & 970.90 & 970.74 & 19.89 & 4.805 & & & 0.330 & & 0.290 \\
\hline & 922.51 & & & 20.13 & 9.266 & & & 5.564 & & \\
\hline \multirow[t]{2}{*}{${ }^{120} \mathrm{Ba}$} & 994.09 & 993.63 & 993.43 & 19.55 & 4.810 & 4.8092 & & 0.320 & & 0.281 \\
\hline & 935.31 & & & 19.51 & 9.494 & & & 5.574 & & \\
\hline \multirow[t]{2}{*}{${ }^{122} \mathrm{Ba}$} & 1015.69 & 1015.50 & 1015.20 & 18.92 & 4.816 & 4.8153 & & 0.321 & 0.345 & 0.273 \\
\hline & 953.92 & & & 18.65 & 9.505 & & & 5.575 & & \\
\hline \multirow[t]{2}{*}{${ }^{124} \mathrm{Ba}$} & 1036.17 & 1036.12 & 1035.98 & 18.31 & 4.822 & 4.8185 & & 0.295 & 0.302 & 0.274 \\
\hline & 978.25 & & & 18.20 & 9.300 & & & 5.577 & & \\
\hline \multirow[t]{2}{*}{${ }^{126} \mathrm{Ba}$} & 1055.66 & 1055.84 & 1055.67 & 17.66 & 4.820 & 4.8221 & & 0.252 & 0.273 & 0.256 \\
\hline & 992.86 & & & 18.46 & 9.293 & & & 5.572 & & \\
\hline \multirow[t]{2}{*}{${ }^{128} \mathrm{Ba}$} & 1074.77 & 1074.68 & 1074.22 & 16.82 & 4.820 & 4.8255 & & 0.215 & 0.249 & 0.218 \\
\hline & 1005.77 & & & 18.71 & 9.306 & & & 5.578 & & \\
\hline \multirow[t]{2}{*}{${ }^{130} \mathrm{Ba}$} & 1093.19 & 1092.72 & 1092.04 & 16.02 & 4.823 & 4.8283 & & 0.181 & 0.218 & 0.171 \\
\hline & 1019.91 & & & 17.27 & 9.338 & & & 5.585 & & \\
\hline \multirow[t]{2}{*}{${ }^{132} \mathrm{Ba}$} & 1110.89 & 1110.04 & 1109.24 & 15.24 & 4.826 & 4.8303 & & 0.143 & 0.186 & 0.143 \\
\hline & 1034.57 & & & 15.69 & 9.351 & & & 5.581 & & \\
\hline \multirow[t]{2}{*}{${ }^{134} \mathrm{Ba}$} & 1127.69 & 1126.70 & 1126.13 & 14.38 & 4.830 & 4.8322 & & 0.101 & 0.161 & -0.113 \\
\hline & 1049.79 & & & 14.39 & 10.48 & & & 5.583 & & \\
\hline
\end{tabular}

\section{DETAILS OF THE CALCULATIONS AND RESULTS}

To-date, there exists a large number of force parameters for finding a convergent solution of the RMF Lagrangian density. In many of our previous works and of others [20, 2224, 32, 34, 35] the ground state properties such as the binding energies (BE), quadrupole deformation parameters $\beta_{2}$, rootmean-square charge radii $\left(r_{c h}\right)$, single-particle level, nucleonic distributions and other bulk properties, are evaluated by using the various parameter sets. From these works, one can conclude that, more or less, most of the recent parameter sets reproduce well the ground state properties, not only of stable normal nuclei but also for exotic nuclei including superheavy. This implies that by using one reasonably acceptable parameter set, the predictions of the model will remain nearly force independent. Here, we have used the most popular NL3 force parameter for this investigation. The numerical calculations are carried out by taking the maximum oscillator quanta $N_{F}=N_{B}=16$ for Fermion and boson. To test the convergence of the solutions, few calculations are done with $N_{F}=N_{B}=12$ also. The variation of these two solutions are $\leq 0.002 \%$ for binding energy and $0.001 \%$ for nuclear radii in drip-line nuclei. Hence, the used model space is good enough for the considered nuclei in a large deformed state. The number of mesh points for Gauss-Hermite and Gauss-Lagurre integration are 20 and 24, respectively. For a given nucleus, as already stated above, the maximum binding energy corresponds to the ground-state, and the other solution with very high deformation is for the fission state.

\section{A. Binding energy, nuclear charge radius and deformation parameter}

To deal with the fission state of a nucleus, it is important as well as necessary to know their ground-state bulk properties, which are mostly responsible for the internal configuration of nuclei. In this context, we first calculated the gross properties such as binding energy, rms charge radius $r_{c h}$ and quadrupole deformation parameter $\beta_{2}$ of Ba-isotopes by using the RMF with NL3 parameter set. The obtained results from RMF(NL3) are compared with the Finite Range Droplet 


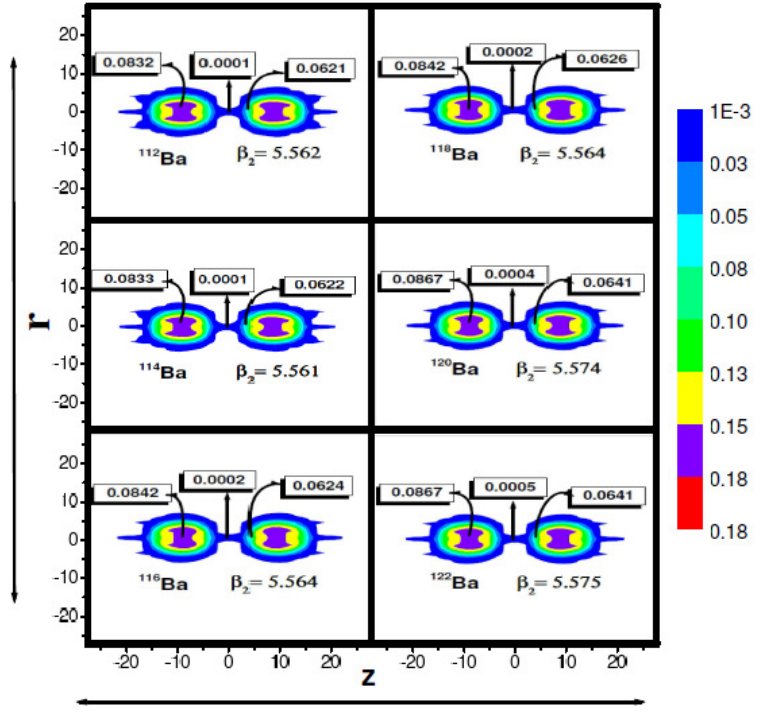

FIG. 1: The neck structure for ${ }^{112-122} \mathrm{Ba}$ in axially deformed coordinates for RMF calculations using NL3 parameter set.

Model (FRDM) predictions [43, 44] and the experimental data [45-47], wherever possible. All the results are listed in Table I. From the table, we find that, in general, the microscopic binding energies, rms charge radii $r_{c h}$ and deformation parameters agree well with the experimental data. The closer comparison of RMF with the FRDM and experimental data shows clearly that the binding energy and radius coincide remarkably well throughout the isotopic chain. However, in case of deformation parameter $\beta_{2}$, the RMF matches the experimental data but not the FRDM predictions. For example, the RMF shows prolate deformed shapes for all ${ }^{122-134} \mathrm{Ba}$ isotopes, which compare nicely with the experimental data but not with the FRDM results, in particular for ${ }^{134} \mathrm{Ba}$ where it is predicted to be oblate in FRDM.

\section{B. The neck configurations of ${ }^{112-134} \mathbf{B a}$}

Generally, the internal configuration of a nucleus can be determined from the density distributions of the nucleons for a given state. The total density of a nucleus (i.e. sum of the neutron and proton densities) in the $\rho z$ plane from RMF(NL3) are obtained for the positive quadrant of the plane parallel to the symmetry $z$-axis. Here, $\rho=x=y=r_{\perp}$ and $z$ is the symmetric axis. It is to be noted that, both the axes $z$ and $\rho$ are conserved in the present formalism under the space reflection symmetry. Now we can obtain the complete picture of a nucleus in the $\rho-z$ plane by reflecting the first quadrant to other successive quadrants. The contour plotting of density along with the color code with corresponding density ranges for the neck structure of the fission state of ${ }^{112-134} \mathrm{Ba}$ are shown in Figs. 1 and 2. From the color code, one can identify the mag-

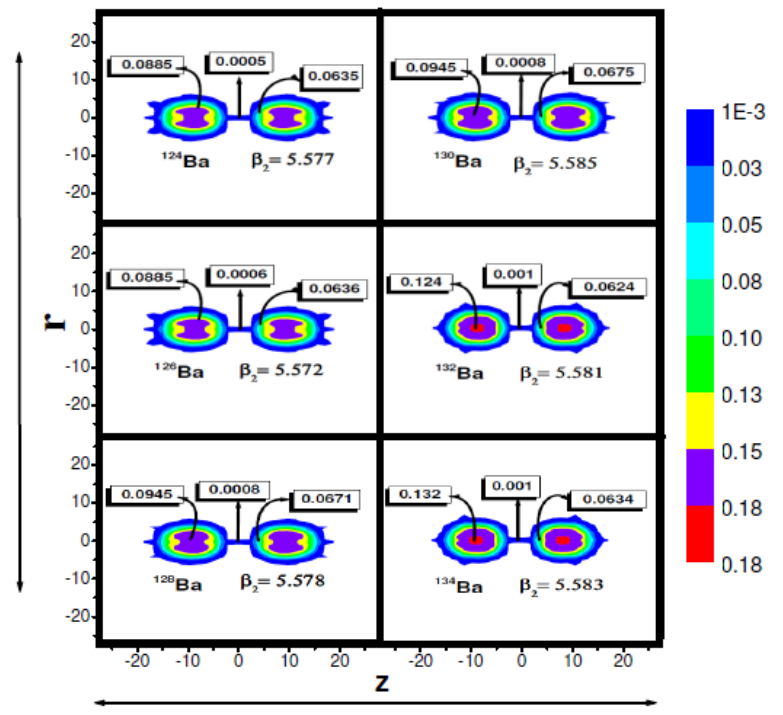

FIG. 2: Same as for Fig. 1, but for ${ }^{124-134} \mathrm{Ba}$.

TABLE II: The RMF(NL3) results for neck configurations of ${ }^{112-134} \mathrm{Ba}$ in the fission state giving the ranges of the neck and their $\mathrm{Z}$ and $\mathrm{N}$ constituents.

\begin{tabular}{cccccc}
\hline \hline Nucleus & $\beta_{2}$ & Range $\left(r_{1}, r_{2} ; z_{1}, z_{2}\right)$ & $Z_{\text {neck }}$ & $N_{\text {neck }}$ & Neck Nucleus \\
\hline${ }^{112} \mathrm{Ba}$ & 5.562 & $( \pm 2.28 ; \pm 1.25)$ & 2.07 & 1.91 & ${ }^{4} \mathrm{He}$ \\
${ }^{114} \mathrm{Ba}$ & 5.561 & $( \pm 2.28 ; \pm 1.25)$ & 2.04 & 2.95 & ${ }^{4} \mathrm{He}$ \\
${ }^{116} \mathrm{Ba}$ & 5.564 & $( \pm 2.27 ; \pm 1.25)$ & 2.05 & 2.03 & ${ }^{4} \mathrm{He}$ \\
${ }^{118} \mathrm{Ba}$ & 5.564 & $( \pm 2.27 ; \pm 1.26)$ & 2.06 & 2.13 & ${ }^{4} \mathrm{He}$ \\
${ }^{120} \mathrm{Ba}$ & 5.574 & $( \pm 2.26 ; \pm 1.26)$ & 2.08 & 2.08 & ${ }^{4} \mathrm{He}$ \\
${ }^{122} \mathrm{Ba}$ & 5.575 & $( \pm 2.26 ; \pm 1.26)$ & 2.05 & 2.18 & ${ }^{4} \mathrm{He}$ \\
${ }^{124} \mathrm{Ba}$ & 5.577 & $( \pm 2.26 ; \pm 1.26)$ & 2.05 & 2.08 & ${ }^{4} \mathrm{He}$ \\
${ }^{126} \mathrm{Ba}$ & 5.572 & $( \pm 2.26 ; \pm 1.27)$ & 2.09 & 2.08 & ${ }^{4} \mathrm{He}$ \\
${ }^{128} \mathrm{Ba}$ & 5.578 & $( \pm 2.26 ; \pm 1.27)$ & 2.11 & 1.98 & ${ }^{4} \mathrm{He}$ \\
${ }^{130} \mathrm{Ba}$ & 5.585 & $( \pm 2.25 ; \pm 1.27)$ & 2.09 & 2.18 & ${ }^{4} \mathrm{He}$ \\
${ }^{132} \mathrm{Ba}$ & 5.581 & $( \pm 2.25 ; \pm 1.27)$ & 2.09 & 2.13 & ${ }^{4} \mathrm{He}$ \\
${ }^{134} \mathrm{Ba}$ & 5.583 & $( \pm 2.25 ; \pm 1.27)$ & 2.08 & 1.12 & ${ }^{4} \mathrm{He}$ \\
\hline \hline
\end{tabular}

nitude of the density range of a particular color code. For example, the color code with deep red corresponds to maximum density $\rho \sim 0.15 \mathrm{fm}^{-3}$ and the olive bearing the minimum value of $\rho \sim 0.001 \mathrm{fm}^{-3}$. (In black and white figures, the color code is read as deep black with maximum density to outer gray as minimum density distribution).

\section{The neck structure with and without pairing}

The pairing is important for open shell nuclei, near and far away from $\beta$-stable region of the nuclear chart. However, for a given nucleus, its value depends slightly on the binding energy 


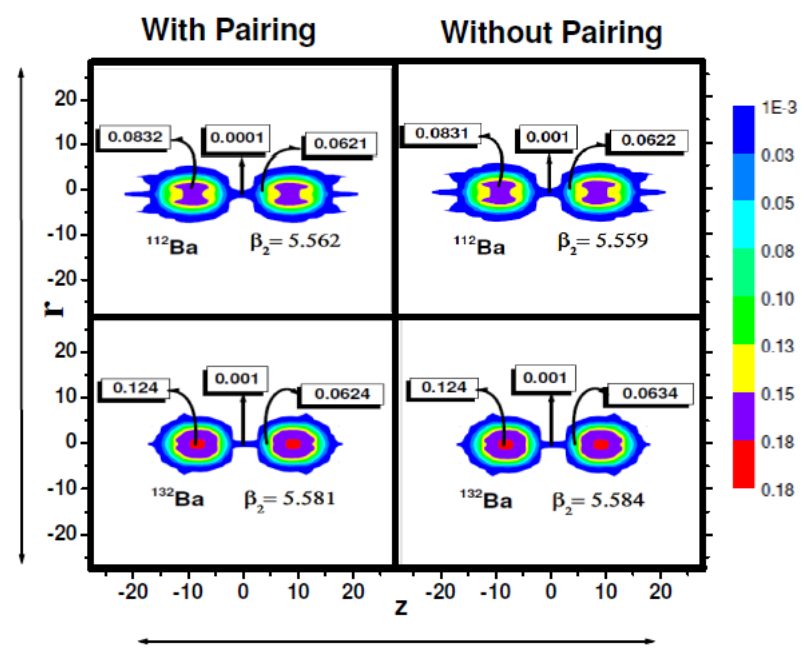

FIG. 3: Same as for Fig. 1, but for ${ }^{112,134} \mathrm{Ba}$, with and without pairing.

and marginally on quadrupole deformation $\beta_{2}$. This means to say that for differing $\beta_{2}$-values in a nucleus, the pairing energy $E_{\text {pair }}$ changes only marginally ( $\sim 2-5 \%$ for this specific region). On the other hand, even if the $\beta_{2}$ values for two nuclei are same, $E_{\text {pair }}$ values are different from one another, depending on the filling of the nucleons. This result is illustrated in Table I for the RMF(NL3) calculation, where pairing energy $E_{\text {pair }}$ for both the ground-state (g.s.) and fission state (f.s.), and their corresponding $\beta_{2}$-values, are displayed. A careful inspection of the pairing energy shows a linear decrease in the magnitude from drip-line to $\beta$-stable line. This trend is also valid for the fission states of these nuclei. For example, the magnitude of pairing energy $E_{\text {pair }}$ for ${ }^{112} \mathrm{Ba}$ and ${ }^{134} \mathrm{Ba}$ are 21 and $14 \mathrm{MeV}$, respectively. The relative difference in the two $E_{\text {pair }}$ values is $7 \mathrm{MeV}$, i.e., the change of $E_{\text {pair }}$ from dripline to $\beta$-stability line is $\sim 30 \%$ for the isotopic chain of $\mathrm{Ba}$. It is well known that the RMF formalism reproduce the cluster structure of nuclei [20-22] which are already predicted by several cluster models [48,-50]. Here, the nucleons are treated as point particles, which oscillates in the mean field of meson medium and gives the way for an independent constraint, resulting in clustering inside the nucleus. On the other hand, one can say that the fluctuations in the central density of the nucleons are due to the shell effects, which may be caused by the pairing correlation of nucleons around the Fermi surface. If this is true, then it would be hard to find the shell gaps and other magic properties in the RMF formalism. Furthermore, we have examined the pairing effect on the neck structure of nuclei. For this purpose, we have plotted the contour of density profiles of ${ }^{112,134} \mathrm{Ba}$ in their fission state, with and without pairing, as the representative cases shown in Fig. 3. From the figure, we found almost identical structures in both the cases. Hence, we one can say that the clustering inside the nuclei is almost independent or very slightly dependent on pairing.

\section{The neck and its constituents}

A careful inspection of the Figs. 1-3 show the formation of a neck structure inside the nucleus, i.e., the preliminary stage of fission. This region has very high decay probability than the other interior part of the nucleus. The most important and crucial attempt made by us here is to see the constituents (neutrons and protons) in this region.

Basically, the constituent of a region depends on the size and the magnitude of the density for that area. To determine the neck structure inside the nucleus, it is important to know the volume of that region, i.e., the ranges of the elongated area in the form of a neck. It is worth mentioning here that the ranges are fixed by graphical method which is guided by the eyes and may have $1-2 \%$ uncertainty in the results. The ranges for the necks of ${ }^{112-134} \mathrm{Ba}$, calculated from Figs. 1-3, are given in Table II. The formula used to identify the ingredient of the neck in the fission state is given by [20-22]:

$$
n=\int_{z_{1}}^{z_{2}} \int_{r_{1}}^{r_{2}} \rho\left(z, r_{\perp}\right) d \tau,
$$

where, $n$ is the number of neutrons $N$ or protons $Z$ or mass $A$, and $z\left(z_{1}, z_{2}\right)$ and $r_{\perp}\left(r_{1}, r_{2}\right)$ are the ranges. From the estimated proton and neutron numbers, we determine the mass number of the nucleus emitted at the time of fission. The obtained nucleons for the fission state, along with the corresponding deformation, for Ba-isotopes are listed in Table II. From the table, we notice the presence of an $\alpha$ (i.e., ${ }^{4} \mathrm{He}$ ) as the residue in $\mathrm{Ba}$-isotopes. Though a straight forward calculation, for the fission state, this is being carried out here for the first time.

\section{SUMMARY AND CONCLUSIONS}

Concluding, we have presented the gross nuclear properties, like the binding energy, deformation parameter $\beta_{2}$, charge radius $r_{c h}$ and the nucleonic density distributions for the isotopic chain ${ }^{112-134} \mathrm{Ba}$, using an axially deformed relativistic mean field formalism with NL3 parameter set. The results of our calculations show a quantitative agreement with the experimental data. We found prolate deformed groundstate solutions for $\mathrm{Ba}$ isotopes, which are consistent with the experimental data but do not match the FRDM predictions for some cases.

Analyzing the total nuclear density distributions, the neck structure, i.e., the fission states of Ba-isotopes are identified. The effect of pairing on the neck structure are taken into account. We find that the neck of Ba-isotopes is built up of ${ }^{4} \mathrm{He}$ nuclei, which can be taken as the evaporation residue of their decay process. Experimentally, the evaporation residue of a decaying compound system consist of multiple neutrons, protons and $\alpha$-particle. It is further noticed that the neck structure of a nucleus remains unaffected for different force parameters, 
as long as the solutions for that nucleus exist. It will be interesting to measure the constituents of evaporation residues of hot Ba-isotopes formed in heavy-ion reactions.
[1] J. A. Wheeler, Phys. Rev. 52, 1107 (1937).

[2] C. F. von Weizssacker, Naturwiss 26, 209 (1938).

[3] W.v Oertzen, M. Freer, Y. Kanada-En'yo, Phys. Rep. 432, 43 (2006), and references therein.

[4] Y. Kanada En'yo, H. Horiuchi, Prog. Theor. Phys. Suppl. 142, 205 (2001).

[5] H. Feldmeier, K. Bieler, J. Schnack, Nucl. Phys. A 586, 493 (1995).

[6] T. Neff, H. Feldmeier, Nucl. Phys. A 713, 311 (2003).

[7] P. Arumugam, B. K. Sharma, S. K. Patra, and R. K. Gupta, Phys. Rev. C 71, 064308 (2005).

[8] R. K. Gupta, S. K. Patra, P. D. Stevenson, C. Beck, and W. Greiner, J. Phys. G: Nucl. Part. Phys. 35, 075106 (2008).

[9] A. Săndulescu, D.N. Poenaru, and W. Greiner, Sovt. J. Part. Nucl, 11, 528 (1980).

[10] D. N. Peonaru, D. Schnabel, W. Greiner, D. Mazilu and R. Gherghescu, At. Data and Nucl. Data Tab., 48, 231, (1991).

[11] H. J. Fink, W. Greiner, R. K. Gupta, S. Liran, H. J. Maruhn, W. Scheid, and O. Zohni, Proc. Int. Conf. on Reactions between Complex Nuclei, Nashville, USA, June 10-14, 1974, ed. R.L. Robinson et. al. (North Holland, Amsterdam 1975), Vol. 2, p. 21

[12] R. K. Gupta, Sovt. J. Part. Nucl. 8, 289 (1977).

[13] S. S. Malik and R. K. Gupta, Phys. Rev. C, 39, 1992, (1989).

[14] H.J. Rose, G.A. Jones, Nature 307, 245 (1984).

[15] H. Matsumura and Y. Suzuki, Nucl. Phys. A, 739, 238, (2004).

[16] T. Wakasa et al., Phys. Lett. B, 653, 173, (2007).

[17] G. L. Zhang, X. Y. Le, Nucl. Phys. A, 848, 292, (2010).

[18] S. Kumar and R. K. Gupta, Phys. Rev. C 49, 1922 (1994).

[19] S. Kumar, D. Bir, and R. K. Gupta, Phys. Rev. C 51, 1762 (1995).

[20] M. Bhuyan, S. K. Patra, P. Arumugam, and Raj K. Gupta, Int. J. Mod. Phys. E, 20, 1227 (2011).

[21] S. K. Patra, R. K. Choudhury and L. Satpathy, J. Phys. G: Nucl. Part. Phys. 37085103 (2010).

[22] M. Bhuyan, Int. J. Mod. Phys. E 22, 1350068 (2013).

[23] S. K. Patra, M. Bhuyan, M. S. Mehta and Raj K. Gupta, Phys. Rev. C 80, 034312 (2009).

[24] M. Bhuyan, S. K. Patra, and Raj K. Gupta, Phys. Rev. C 84, 014317 (2011).

[25] B. Behera, X. Vinas, M. Bhuyan, T. R. Routray, B. K. Sharma, and S. K. Patra, J. Phys. G: Nucl. Part. Phys. 40, 095105 (2013).

[26] E. Bonnet et al., Int. J. Mod. Phys. E 17 (2008) 2359;

[27] G. Ademard et al., Phys. Rev. C 83, 054619 (2011).
[28] B. D. Serot and J. D. Walecka, Adv. Nucl. Phys., 16, 1, (1986). [29] J. Boguta and A. R. Bodmer, Nucl. Phys. A, 292, 413, (1977).

[30] C. J. Horowitz and B. D. Serot, Nucl. Phys. A, 368, 503, (1981).

[31] J. Boguta, H. Stöcker, Phys. Lett. B, 120, 289, (1983).

[32] S. K. Patra and C. R. Praharaj, Phys. Rev. C, 44, 2552, (1991).

[33] W. Pannert, P. Ring, and J. Boguta, Phy. Rev. Lett., 59, 2420, (1986).

[34] Y. K. Gambhir, P. Ring, and A. Thimet, Ann. Phys. (NY) 198, 132 (1990).

[35] G. A. Lalazissis, J. Konig and P. Ring, Phys. Rev. C. 55, (1997) 1.

[36] K. Rutz, J. A. Maruhn, P.-G. Reinhard and W. Greiner, Nucl. Phys. A 590 (1995) 680.

[37] M. Bender, K. Rutz, P.-G. Reinhard, J. A. Maruhn and W. Greiner, Phys. Rev. C 58 (1998) 2126.

[38] P.-G. Reinhard and J. A. Maruhn, Heavey Element and Related Phenomena, Edited by W. Greiner and R. K. Gupta, World Scientific Pub., Vol. 1, Ch. 8 (1999), p. 332.

[39] D. G. Madland and J. R. Nix, Nucl. Phys. A 476, 1 (1981).

[40] S. K. Patra, M. Del Etal, M. Centelles, and X. Vinas, Phy. Rev. C 63, 024311 (2001).

[41] G. A. Lalazissis, D. Vretenar, and P. Ring, Nucl. Phys. A, 650, 133 (1999).

[42] G. A. Lalazissis, D. Vretener, P. Ring, M. Stoitsor and L. M. Robledo, Phys. Rev. C, 60, 014310 (2001).

[43] P. Möller, J. R. Nix, W. D. Myers and W. J. Swiatecki, Atomic and Nucl. Data Tables 59 (1995) 185

[44] P. Möller, J. R. Nix and K. -L. Kratz, Atomic and Nucl. Data Tables 66 (1997) 131.

[45] M. Wang, G. Audi, A. H. Wapstra, F. G. Kondev, M. MacCormick, X. Xu and B. Pfeiffer, Chinese Phys. C, 361603 (2013).

[46] I. Angeli, K.P. Marinova, Atomic Data and Nucl. Data Tables 9969 (2013).

[47] S. Raman, C. W. Nestor, JR., and P. Tikkanen, Atomic Data and Nucl. Data Tables 78, 1 (2001)

[48] A. Tohsaki, H. Horiuchi, P. Chuck, and G. Ropke, Phys. Rev. Lett. 87, 192501 (2001).

[49] Y. Funaki, A. Tohsaki, H. Horiuchi, P. Schuck, and G. Ropke, Phys. Rev. C, 67, 051306 (R) (2003).

[50] Y. Funaki, A. Tohsaki, H. Horiuchi, P. Schuck, and G. Ropke, Mod. Phys. Lett. A, 21, 2331 (2006). 